\title{
FRACTIONAL BROWNIAN MARKETS WITH TIME-VARYING VOLATILITY AND HIGH-FREQUENCY DATA
}

\author{
Ananya Lahiri ${ }^{1, \dagger}$ and Rituparna Sen $^{2}$ \\ ${ }^{1}$ Chennai Mathematical Institute, Chennai, India \\ ${ }^{2}$ Indian Statistical Institute, Chennai, India
}

\begin{abstract}
Diffusion processes driven by Fractional Brownian motion (FBM) have often been considered in modeling stock price dynamics in order to capture the long range dependence of stock price observed in reality. Option prices for such models had been obtained by Necula (2002) under constant drift and volatility. We obtain option prices under time varying volatility model. The expression depends on volatility and the Hurst parameter in a complicated manner. We derive a central limit theorem for the quadratic variation as an estimator for volatility for both the cases, constant as well as time varying volatility. That will help us to find estimators of the option prices and to find their asymptotic distributions.

Keywords: asymptotic normality, fractional Black Scholes model, Malliavin calculus, option price, volatility, Wick financing, Wick Ito Skorohod integration, Wiener chaos.
\end{abstract}

\section{Introduction}

It has been proposed to model stock prices as a diffusion driven by fractional Brownian motion (fBm) in order to capture long range dependence of stock price in reality. See Cont (2005) for evidence of long memory in finance and relation to fractional Brownian motion.

Cheridito (2003) has shown that the solution of the diffusion equation driven by fBm with suitably time lag will lead to an arbitrage-free model. Guasoni (2006) has shown no arbitrage under transaction cost for fBm model. Elliott and Van der Hoek (2003), Biagini et al. (2004) have shown under Wick Ito Skorohod notion of integration one can get arbitrage free market with $\mathrm{fBm}$ in some sense. Option prices for such models are obtained by Necula (2002) under constant drift and volatility. One of the aims of this paper is to obtain estimator for some functional of volatility which can be used to price option under time varying volatility model.

For Brownian motion (Bm) setup, the estimation of one of the important functional of volatility appeared in option price formula, called integrated volatility, is performed using sum of frequently sampled squared data. For high frequency data with equal interval this estimator is essentially quadratic variation. FBm is long memory process for Hurst parameter $H \in\left(\frac{1}{2}, 1\right)$. It is well established result that for pure $\mathrm{fBm}$ with $H \in\left(\frac{1}{2}, \frac{3}{4}\right)$ quadratic variation is asymptotically normal. Using that result we will show that in the diffusion driven by $\mathrm{fBm}$ with $H<\frac{3}{4}$ with constant volatility, quadratic variation is asymptotically normal. For similar model and low frequency data with fixed time gap asymptotic normality for volatility estimator was obtained by Xiao et al.(2013). In our paper we consider the estimator for high frequency data with time intervals decreasing to zero and show the asymptotic normality for the estimator. Confidence intervals for volatility can now be translated to confidence intervals for option prices as the expression for option price involve the quantity volatility. For diffusion driven by fBm with $H \in\left(\frac{1}{2}, \frac{3}{4}\right)$ and time varying bounded volatility case also we will show the asymptotic normality of the estimator from high frequency data.

The objective of this paper is two fold. Firstly for the diffusion driven by fBm with time varying volatility we will find the option price in terms of some functional of volatility and the Hurst parameter. Secondly we will show the asymptotic normality property for the estimator for such parametric

†Corresponding author, E-mail: ananya.isi@gmail.com. 
function. The estimator requires the prior knowledge of Hurst parameter. Once the estimate of functional of volatility is found one can apply the estimate to get the option price.

The rest of the paper is organized as follows. In section 2 we describe the diffusion model for the stock price. In section 3 we introduce the proper notion of integration required to obtain an arbitrage-free solution of the diffusion equation. In section 4 we present the option pricing results. The central limit theorems for the proposed estimator are obtained in section 5. We conclude and summarize the current and future research directions in section 6 .

\section{Model}

The introduction of the fractional Black-Scholes model, where the Bm in the classical Black-Scholes model is replaced by a fBm, have been motivated by empirical studies (see for example Mandelbrot (1997), Shiryaev (1999)). The risk free asset equation is

$$
d P_{t}=r_{t} P_{t} d t, \quad P_{0}=1, \quad 0 \leq t \leq T
$$

The risky asset equation is

$$
d S_{t}=\mu_{t} S_{t} d t+\sigma_{t} S_{t} d B_{t}^{H}, \quad S_{0}=S>0, \quad 0 \leq t \leq T
$$

where $B_{t}^{H}$ is FBM with initial condition $S_{0}=S>0$. Here $H$ is Hurst parameter, for $0<H<1$. $\mu_{t}$ is real valued deterministic function of time $t$, called drift and $\sigma_{t}^{2}$ is positive real valued deterministic function of $t$, called volatility. $B_{t}^{H}$ is a continuous and centered Gaussian process starting at 0 with covariance and variance functions as follows: $\forall H \in(0,1), s, t>0$

$$
\begin{gathered}
\mathrm{E}\left(B_{t}^{H} B_{s}^{H}\right)=\frac{1}{2}\left(t^{2 H}+s^{2 H}-|t-s|^{2 H}\right) \\
E\left(B_{t}^{H}\right)^{2}=t^{2 H},
\end{gathered}
$$

$B_{t}^{H}$ has homogeneous increments, i.e., $B_{t+s}^{H}-B_{s}^{H}$ has same law as $B_{t}^{H}$ for all $s, t>0$. Increments are dependent and correlation between the increments $B_{t+h}^{H}-B_{t}^{H}$ and $B_{s+h}^{H}-B_{s}^{H}$ with $s+h \leq t$ and $t-s=n h$ is as follows:

$$
\rho_{n}^{H}=\frac{1}{2} h^{2 H}\left[(n+1)^{2 H}+(n-1)^{2 H}-2 n^{2 H}\right]
$$

Firstly we will find the European call option price for this model. Secondly we try to provide an estimator for option price. In this process we see that it is enough to study the quadratic variation of this process for given high frequency data $0=t_{0}<t_{1}<\cdots<t_{N}=1$ with $S_{t_{j}}, j=0, \cdots, N$ and $t_{j+1}-t_{j}=\frac{1}{N} \forall j=0, \cdots, N-1$. We will propose suitable estimator with this data and see where it converges and how that is useful for estimating option price. We observe that the analysis is based on high frequency data, as sample size increases the time difference between two consecutive data point decreases. We also note that through out our analysis we know $H$, we do not estimate $H$ from data.

\section{Regarding the solution of the SDE, Wick Ito Skorohod Integral, $H \in(0,1)$}

In order to find the solution of the diffusion equation (2.2), we need to note that the whole analysis depends on how we interpret the term $d B_{t}^{H}$. For $H \neq 1 / 2, B_{t}^{H}$ is not a semimartingale.

There are different notions of integration with respect to $B_{t}^{H}$, out of which we choose Wick Ito Skorohod (WIS) integral notion to solve equation (2.2), for $H \in(0,1)$, due to financial reason outlined in, for example, see Elliot and Van der Hoek (2003) and Biagini et.al. (2004),(2008).

Rogers (1997) explains why other common notions of integral are inappropriate.

Following the WIS notion of integration the solution of the stochastic differential equation (2.2) is

$$
S_{t}=S_{0} \exp \left(\int_{0}^{t} \sigma_{s} d B_{s}^{H}+\int_{0}^{t} \mu_{s} d s-\frac{1}{2} \int_{\mathbb{R}}\left(M\left(\sigma_{s} \chi_{[0, t]}\right)\right)^{2} d s\right)
$$


where $M$ is an operator acting on $s$ and depends on $H ; \chi_{[0, t]}$ is the indicator function. See Biagini et al. $(2004,2008)$. We will discuss about the operator $M$ in next subsection and meaning of $\int_{0}^{t} \sigma_{s} d B_{s}^{H}$ in following subsection.

3.1. The integral operator $M$. Let us elaborate about the operator $M$ which will be needed in future sections, See Biagini et al. (2004). Let $\mathcal{S}(\mathbb{R})$ denote the Schwartz space of smooth rapidly decreasing functions on $\mathbb{R}$. $M$ is defined on $\mathcal{S}(\mathbb{R})$ to $L^{2}(\mathbb{R})$ as follows:

$$
M f(x)=-\frac{d}{d x} C_{H} \int_{\mathbb{R}}(t-x)|t-x|^{H-\frac{3}{2}} f(t) d t
$$

$C_{H}$ is constant.

$$
M f(y)=|y|^{\frac{1}{2}-H} \hat{f}(y), y \in \mathbb{R}
$$

with Fourier transform $\hat{f}$ defined as

$$
\hat{f}(y)=\int_{\mathbb{R}} e^{-i x y} f(x) d x .
$$

It turns out that $C_{H}=\frac{[\Gamma(2 H+1) \sin (\pi H)]^{\frac{1}{2}}}{\left[2 \Gamma\left(H-\frac{1}{2}\right) \cos \left(\frac{1}{2} \pi\left(H-\frac{1}{2}\right)\right)\right]}, \Gamma($.$) is gamma function and explicit expression$ for $M$ is as follows:

$$
\begin{aligned}
& \text { for } H \in\left(0, \frac{1}{2}\right), M f(x)=C_{H} \int_{\mathbb{R}} \frac{f(x-t)-f(x)}{|t|^{\frac{3}{2}-H}} d t \\
& \qquad \text { for } H=\frac{1}{2}, M f(x)=f(x) \\
& \text { for } H \in\left(\frac{1}{2}, 1\right), M f(x)=C_{H} \int_{\mathbb{R}} \frac{f(t)}{|t-x|^{\frac{3}{2}-H}} d t
\end{aligned}
$$

$M$ extends $\mathcal{S}(\mathbb{R})$ to $L_{H}^{2}$ where

$$
\begin{aligned}
L_{H}^{2}(\mathbb{R}) & =\left\{f: \mathbb{R} \rightarrow \mathbb{R}(\text { deterministic }) ;|y|^{\frac{1}{2}-H} \hat{f}(y) \in L^{2}(\mathbb{R})\right\} \\
& =\left\{f: \mathbb{R} \rightarrow \mathbb{R} ; M f(x) \in L^{2}(\mathbb{R})\right\} \\
& \left.=\left\{f: \mathbb{R} \rightarrow \mathbb{R} ;\|f\|_{L_{H}^{2}}<\infty\right\}, \text { where }\|f\|_{L_{H}^{2}}=\|M f\|_{L^{2}}\right\}
\end{aligned}
$$

We also have for $f \in L_{H}^{2}(\mathbb{R})$

$$
\langle f, g\rangle_{L_{H}^{2}}=\langle M f, M g\rangle_{L^{2}}
$$

and for $f, g \in L^{2}(\mathbb{R}) \cap L_{H}^{2}(\mathbb{R})$

$$
\langle f, M g\rangle_{L^{2}}=\langle\hat{f}, \widehat{M g}\rangle_{L^{2}}=\langle\widehat{M f}, \hat{g}\rangle_{L^{2}}=\langle M f, g\rangle_{L^{2}}
$$

3.2. Wiener Integral with respect to FBM, $H \in(0,1)$. Let $f \in L_{H}^{2}(\mathbb{R})$, deterministic. Then $M f \in L^{2}(\mathbb{R})$. The Wiener integral with respect to fractional Brownian motion is defined as

$$
\int_{\mathbb{R}} f(s) d B_{s}^{H}=\int_{\mathbb{R}}(M f)(s) d B_{s}
$$

For detail see Appendix.

\section{On the way to calculate European call option price}

In this section we follow similar line of argument in that of Elliot and Van der Hoek (2003). 
4.1. Risk-neutral measure. Let us write equation (2.2) with the notion if Wick product. Now following Elliott and Van der Hoek (2003) we rewrite equation (2.2) as follows:

$$
d S_{t}=S_{t} \diamond\left[\mu_{t}+\sigma_{t} W_{t}^{H}\right] d t
$$

where $\diamond$ is the Wick product for two processes and $W_{t}^{H}=\frac{d B_{t}^{H}}{d t}$. For meaning of Wick product and $W_{t}^{H}$ see Appendix (8.2). From theorem of "Wick Ito integral" Biagini et al. (2008) or (Appendix (8.2)) we note that $S_{t} d B_{t}^{H}=S_{t} \diamond W_{t}^{H} d t$. We denote trading strategy or portfolio as $\theta(t, \omega)=\theta(t)=$ $(u(t), v(t))=\left(u_{t}, v_{t}\right)$ where $u(t)$ and $v(t)$ are the number of units of bond and stock respectively in the portfolio at time $t$ and the processes are adaptive. The value process is defined as

$$
z_{t}^{\theta}=u_{t} P_{t}+v_{t} S_{t}
$$

Definition: The concept analogous to self-financing in the fractional Brownian setting is Wickfinancing. Elliott and Van der Hoek mention it as self financing but Wick financing strategy is not usual buy and hold strategy. A portfolio is Wick-financing if

$$
\begin{aligned}
d z_{t}^{\theta}= & u_{t} d P_{t}+v_{t} d S_{t}=u_{t} d P_{t}+v_{t} S_{t} \diamond\left[\mu_{t}+\sigma_{t} W_{t}^{H}\right] d t \\
d z_{t}^{\theta} & =u_{t} d P_{t}+v_{t} S_{t} \diamond\left(\mu_{t}+\sigma_{t} W_{t}^{H}\right) d t \\
& =u_{t} r_{t} P_{t} d_{t}+v_{t} S_{t} \diamond \mu_{t} d t+\sigma_{t} v_{t} S_{t} \diamond W_{t}^{H} d t \\
& =\left(z_{t}-v_{t} S_{t}\right) r_{t} d_{t}+\mu_{t} v_{t} S_{t} d t+\sigma_{t} v_{t} S_{t} \diamond W_{t}^{H} d t \\
& =z_{t} r_{t} d t+\sigma_{t} v_{t} S_{t} \diamond\left(\frac{\mu_{t}-r_{t}}{\sigma_{t}}+W_{t}^{H}\right) d t
\end{aligned}
$$

From the Girsanov theorem in Elliott and Van der Hoek (2003) the translated process $\hat{B}_{t}^{H}$ as

$$
\hat{B}_{t}^{H}=\int_{0}^{t} \frac{\mu_{s}-r_{s}}{\sigma_{s}} d_{s}+B_{t}^{H}
$$

is fBm with respect to new measure $\hat{P}$ defined on $\mathcal{F}$ by $\frac{d \hat{P}}{d P}=\exp \left(\langle\omega, \phi\rangle-\frac{1}{2}\|\phi\|_{L^{2}}^{2}\right)=\exp \left(\int_{\mathbb{R}} \phi(s) d B_{s}-\right.$ $\left.\frac{1}{2}\|\phi\|_{L^{2}}^{2}\right)$ where $\phi(s)=M^{-1}\left(\frac{r(s)-\mu(s)}{\sigma(s)}\right) I_{[0, t]}(s)$. For notation $\exp \left(\langle\omega, \phi\rangle-\frac{1}{2}\|\phi\|_{L^{2}}^{2}\right)$ see Appendix (8.1). We note that $\phi(s)$ has to be in $L^{2} \mathbb{R}$.

Now we can rewrite 4.3 as

$$
d z_{t}=r_{t} z_{t} d t+\sigma_{t} v_{t} S_{t} \diamond \hat{W}_{t}^{H} d t
$$

where, $\hat{W}_{t}^{H}=\frac{d \hat{B}_{t}^{H}}{d t}$. Multiplying both sides with $\exp \left(\tilde{r}_{t}\right)$ with $\tilde{r}_{t}=\int_{0}^{t} r_{s} d s$ and integrating, we get

$$
e^{-\tilde{r}_{t}} z_{t}-z_{0}=\int_{0}^{t} e^{-\tilde{r}_{s}} \sigma_{s} v_{s} S_{s} \diamond \hat{W}_{s}^{H} d s
$$

and

$$
\hat{E}\left[e^{-\tilde{r}_{T}} z_{T}\right]=z_{0}
$$

where $\hat{E}$ is expectation under measure $\hat{P}$. Thus there exists a risk-neutral measure.

We note that under risk neutral measure $\hat{P}$ we have

$$
d S_{t}=r_{t} S_{t} d t+\sigma_{t} S_{t} d \hat{B}_{t}^{H}
$$

which will be useful for calculating option price. 
4.2. Complete Market. Let $\mathcal{F}_{t}=\sigma\left\{B_{s}^{H}, 0 \leq s \leq t\right\}$ be the filtration. The market is complete if $\forall \mathcal{F}_{T}$ measurable bounded random variable $F, \exists z \in \mathbb{R}$ and portfolio $\left(u_{t}, v_{t}\right)$ such that $F=z_{T}$ almost surely $\hat{P}$, where $z_{T}$ is given by 4.2 . We now proceed to verify this. By fractional Clark-Ocone theorem in Elliott and Van der Hoek (2003) applied to $F$, we have,

$$
e^{-\tilde{r}_{T}} F=\hat{E}\left[e^{-\tilde{r}_{T}} F\right]+\int_{0}^{T} \tilde{E}_{\hat{P}}\left[\hat{D}_{t}\left(e^{-\tilde{r}_{T}} F\right) \mid \mathcal{F}_{t}\right] \diamond \hat{W}_{t}^{H} d t
$$

Here $\tilde{E}_{\hat{P}}$ denotes the quasi-conditional expectation and $\hat{D}_{t}$ is the fractional Hida Malliavin derivative with respect to $\hat{B}_{t}^{H}$. For detail see Elliot and van der Hoek (2003)and Biagini et. al. (2008). We take $z=\hat{E}\left[e^{-\tilde{r}_{T}} F\right]$. Now comparing equations (4.6) and (4.8) we get

$$
\tilde{E}_{\hat{P}}\left[\hat{D}_{t}(F) \mid \mathcal{F}_{t}\right]=e^{\tilde{r}_{T}-\tilde{r}_{t}} \sigma_{t} v_{t} S_{t}
$$

This is the condition for completeness of the market. Here we note that there is criticism about this notion of completeness with Wick financing instead of self financing, see Bjrk and Hult (2005). Fractional Black Scholes market has weak arbitrage but no strong arbitrage, see Biagini et al.(2008).

In the context of quasi conditional expectation we require following lemma which will be useful for calculating option price in next section.

Lemma 4.1. a) If $g_{t}=\exp \left(\int_{0}^{t} \sigma_{s} d \hat{B}_{s}^{H}-\frac{1}{2} \int_{\mathbb{R}}\left(M\left(\sigma_{s} \chi_{[0, t]}\right)\right)^{2} d s\right)$ then for $T>t, \tilde{E}_{\hat{P}}\left[g_{T} \mid \mathcal{F}_{t}\right]=g_{t}$.

b) If $F \in L^{1,2}(\hat{P})$ (similar to Definition A4 of Elliott and Van der Hoek (2003)), and $G_{t}=$ $\int_{0}^{t} F_{t} d \hat{B}_{t}^{H}$, then for $T>t, \tilde{E}_{\hat{P}}\left[G_{T} \mid \mathcal{F}_{t}\right]=G_{t}$.

Proof. Proof can be done by direct calculation.

4.3. Price of European Call Option. We next will find the European call option price $\tilde{E}_{\hat{P}}\left[\left(S_{T}-\right.\right.$ $\left.K)_{+} \mid \mathcal{F}_{t}\right]$ for this model. When $\mu_{t}=\mu$ and $\sigma_{t}=\sigma$, Necula (2008) obtains the price $C$ at every $t \in(0, T)$ of an European call option with strike price $K$ and maturity $T$ as

$$
\begin{aligned}
& C\left(t, S_{t}\right)=S_{t} \Phi\left(d_{1}\right)-K e^{-r(T-t)} \Phi\left(d_{2}\right) \\
& \text { where } \quad d_{1}=\frac{\log \left(\frac{S_{t}}{K}\right)+r(T-t)+\frac{\sigma^{2}}{2}\left(T^{2 H}-t^{2 H}\right)}{\sigma \sqrt{T^{2 H}-t^{2 H}}} \quad \text { and } \\
& d_{2}=\frac{\log \left(\frac{S_{t}}{K}\right)+r(T-t)-\frac{\sigma^{2}}{2}\left(T^{2 H}-t^{2 H}\right)}{\sigma \sqrt{T^{2 H}-t^{2 H}}}
\end{aligned}
$$

and $\Phi()$ is the cumulative probability of the standard normal distribution.

The confidence intervals for $\sigma^{2}$ obtained in section 5 can be translated to prediction intervals for $C$ as in Mykland (2000) or Avellaneda et al (1995).

Next for time varying $\mu_{t}$ and $\sigma_{t}$ let us calculate option price. We need the following lemma.

Lemma 4.2. The price at every $t \in[0, T]$ of bounded $\mathcal{F}_{T}$ measurable function $F \in L^{2}(\hat{P})$ is given by $F(t)=\exp \left(-\tilde{r}_{T}+\tilde{r}_{t}\right) \tilde{E}_{\hat{P}}\left[F \mid \mathcal{F}_{t}\right]$.

Proof. Proof can be followed in similar line as in Theorem 4.1 from Necula (2008) and using part b) of lemma 4.1.

For European call option price $F$ will be $F(\omega)=(S(T, \omega)-K)^{+}$where $K$ is the strike price.

Now following similar line of approach from Theorem 3.1 of Necula (2008) and using part a) of lemma 4.1 we get

$$
\tilde{E}_{\hat{P}}\left(\exp \left(\int_{0}^{T} \sigma_{s} d \hat{B}_{s}^{H}\right) \mid \mathcal{F}_{t}\right)=\exp \left(\int_{0}^{t} \sigma_{s} d \hat{B}_{s}^{H}-\frac{1}{2} \int_{\mathbb{R}}\left(M\left(\sigma_{s} \chi_{[0, t]}\right)\right)^{2} d s+\frac{1}{2} \int_{\mathbb{R}}\left(M\left(\sigma_{s} \chi_{[0, T]}\right)\right)^{2} d s\right)
$$

Equation (4.12) will be used for proving next theorem. 
Theorem 4.1. The price at every $T \in[0, T]$ of an European call option with strike price $K$ and maturity $T$ is given by $S_{t} \Phi\left(d_{1}\right)-K \exp \left(-\tilde{r}_{T}+\tilde{r}_{t}\right) \Phi\left(d_{2}\right)$ where

$$
d_{1}=\frac{\ln \left(S_{t} / K\right)+\tilde{r}_{T}-\tilde{r}_{t}+\int_{\mathbb{R}}\left(M\left(\sigma_{s} \chi_{[0, T]}\right)\right)^{2} d s-\int_{\mathbb{R}}\left(M\left(\sigma_{s} \chi_{[0, t]}\right)\right)^{2} d s}{\sqrt{\int_{\mathbb{R}}\left(M\left(\sigma_{s} \chi_{[0, T]}\right)\right)^{2} d s-\int_{\mathbb{R}}\left(M\left(\sigma_{s} \chi_{[0, t]}\right)\right)^{2} d s}}
$$

and

$$
d_{2}=\frac{\ln \left(S_{t} / K\right)+\tilde{r}_{T}-\tilde{r}_{t}-\int_{\mathbb{R}}\left(M\left(\sigma_{s} \chi_{[0, T]}\right)\right)^{2} d s+\int_{\mathbb{R}}\left(M\left(\sigma_{s} \chi_{[0, t]}\right)\right)^{2} d s}{\sqrt{\int_{\mathbb{R}}\left(M\left(\sigma_{s} \chi_{[0, T]}\right)\right)^{2} d s-\int_{\mathbb{R}}\left(M\left(\sigma_{s} \chi_{[0, t]}\right)\right)^{2} d s}}
$$

Proof. Proof is in similar line as that of given in Necula (2008).

\section{Estimation of volatility from discrete observations}

Assume that the process is observed at discrete-time instants $0=t_{0}<t_{1}<t_{2}<\cdots<t_{N}=T$. Thus the observation vector is $S=\left(S_{t_{1}}, S_{t_{2}}, \cdots, S_{t_{N}}\right)$. We note that this is high frequency data. In particular, we assume $t_{k}=k h, k=1,2, \cdots, N$ for a step size, $h=\frac{1}{N}>0$. In section 5.1 we present the results when $\sigma_{t}$ is constant and in section 5.3 when $\sigma_{t}$ is time varying. In section 5.2 and 5.4 we present simulation studies.

5.1. Constant $\sigma$. Let us start with the estimator of $\sigma^{2}$ as

$$
\hat{\sigma^{2}}=\frac{1}{N h^{2 H}} \sum_{k=0}^{N-1}\left(\log \left(S_{t_{k+1}}\right)-\log \left(S_{t_{k}}\right)\right)^{2}
$$

We shall prove a central limit theorem for $\hat{\sigma}^{2}$. The main component of the proof is central limit theorem for quadratic variations of fractional Brownian motion. We also need to bound the additional terms that comes from the geometric nature of our process. Some of these arguments are similar to those of Nourdin $(2008,2009)$. The main theorem for this section is given below.

Theorem 5.1. Assume that the stock price follows the diffusion model specified by equation (4.4) with no drift and constant volatility $\sigma$. Also assume that $H \in(0,3 / 4) . N \rightarrow \infty$ with the observation interval $N h=T$ remaining constant. Without loss of generality we can assume $T=1$. Then

$$
\sqrt{N}\left(\hat{\sigma^{2}}-\sigma^{2}\right) \Longrightarrow \mathcal{N}\left(0, \sigma_{H, 2}^{2}\right)
$$

where $\sigma_{H, 2}^{2}$ is a constant that can be computed explicitly,

$$
\sigma_{H, 2}^{2}=2 \sigma^{4} \lim _{N \rightarrow \infty}\left(1+2\left(1-\frac{1}{N}\right)\left(2^{2 H}-1\right)^{2}+\sum_{k=2}^{N}\left(1-\frac{k}{N}\right)\left[(k+1)^{2 H}+(k-1)^{2 H}-2 k^{2 H}\right]^{2}\right) .
$$

Proof. Under the condition of $\mu=0$ and $\sigma_{t}=\sigma$, the solution (3.1) of the stochastic differential equation (4.2) simplifies to

$$
S_{t}=S_{0} \exp \left(\sigma d B_{t}^{H}-\frac{1}{2} \sigma^{2} t^{2 H}\right) .
$$

Putting this solution in the definition of $\hat{\sigma}^{2}$ in equation (5.1), we get,

$$
\begin{aligned}
\hat{\sigma^{2}}=\frac{1}{N h^{2 H}} \sum_{k=0}^{N-1}\left[\sigma\left(B_{(k+1) h}^{H}-B_{k h}^{H}\right)-\frac{\sigma^{2}}{2}\left(\{(k+1) h\}^{2 H}-\{k h\}^{2 H}\right)\right]^{2} \\
=\frac{1}{N h^{2 H}} \sigma^{2}\left[\begin{array}{l}
\sum_{k=0}^{N-1}\left(B_{(k+1) h}^{H}-B_{k h}^{H}\right)^{2} \\
-\sigma \sum_{k=0}^{N-1}\left\{\left(B_{(k+1) h}^{H}-B_{k h}^{H}\right)\left(\{(k+1) h\}^{2 H}-\{k h\}^{2 H}\right)\right\} \\
+\frac{\sigma^{2}}{4} \sum_{k=0}^{N-1}\left(\{(k+1) h\}^{2 H}-\{k h\}^{2 H}\right)^{2}
\end{array}\right]
\end{aligned}
$$


It is already known, for example putting $\kappa=2$ in equation (1.5) of Nourdin(2008), that if $H \in$ $(0,3 / 4)$,

$$
X_{N}:=\frac{1}{\sqrt{N}} \sum_{k=0}^{N-1}\left[h^{-2 H}\left(B_{(k+1) h}^{H}-B_{k h}^{H}\right)^{2}-1\right] \Longrightarrow \mathcal{N}\left(0, \sigma_{H, 2}^{2}\right)
$$

Combining (5.4) and (5.5), we have

$$
\begin{aligned}
\sqrt{N}\left(\hat{\sigma^{2}}-\sigma^{2}\right)= & X_{N}-\frac{\sigma^{3}}{\sqrt{N} h^{2 H}} \sum_{k=0}^{N-1}\left\{\left(B_{(k+1) h}^{H}-B_{k h}^{H}\right)\left(\{(k+1) h\}^{2 H}-\{k h\}^{2 H}\right)\right\} \\
& +\frac{\sigma^{4}}{4 \sqrt{N} h^{2 H}} \sum_{k=0}^{N-1}\left(\{(k+1) h\}^{2 H}-\{k h\}^{2 H}\right)^{2} .
\end{aligned}
$$

It is shown in lemma 5.1 that the second term converges to zero in $L^{2}$ as $N \rightarrow \infty$. In lemma 5.2 it is shown that the third term converges to zero. The theorem now follows by applying Chebyshev's inequality to get convergence in $L^{2}$ implies convergence in probability and Slutsky's theorem to get final asymptotic normality.

Lemma 5.1. Under the assumptions of theorem 5.1,

$$
\frac{\sigma^{3}}{\sqrt{N} h^{2 H}} \sum_{k=0}^{N-1}\left\{\left(B_{(k+1) h}^{H}-B_{k h}^{H}\right)\left(\{(k+1) h\}^{2 H}-\{k h\}^{2 H}\right)\right\} \stackrel{L^{2}}{\longrightarrow} 0
$$

Proof. As $h=\frac{1}{N}$ l.h.s of (5.7) is

$$
U_{1}=\sigma^{3} N^{2 H-\frac{1}{2}} \sum_{k=0}^{N-1}\left(B_{\frac{k+1}{N}}^{H}-B_{\frac{k}{N}}^{H}\right)\left(\left(\frac{k+1}{N}\right)^{2 H}-\left(\frac{k}{N}\right)^{2 H}\right)
$$

Now we have $E\left(B_{\frac{k+1}{N}}^{H}-B_{\frac{k}{N}}^{H}\right)^{2}=\frac{1}{N^{2 H}}$

$$
\begin{gathered}
E\left(B_{\frac{k+1}{N}}^{H}-B_{\frac{k}{N}}^{H}\right)\left(B_{\frac{k+2}{N}}^{H}-B_{\frac{k+1}{N}}^{H}\right)=\frac{2^{2 H}-1}{N^{2 H}} \sim \frac{1}{N^{2 H}} \\
E\left(B_{\frac{k+1}{N}}^{H}-B_{\frac{k}{N}}^{H}\right)\left(B_{\frac{j+1}{N}}^{H}-B_{\frac{j}{N}}^{H}\right)=\frac{1}{2}\left[\left|\frac{k-j+1}{N}\right|^{2 H}+\left|\frac{k-j-1}{N}\right|^{2 H}-2\left|\frac{k-j}{N}\right|^{2 H}\right] \text { for }|k-j|>1 .
\end{gathered}
$$

Then second moment of (5.8) becomes

$$
\begin{gathered}
\sigma^{6} N^{4 H-1} \sum_{k=0}^{N-1} \sum_{j=0}^{N-1}\left(\left(\frac{k+1}{N}\right)^{2 H}-\left(\frac{k}{N}\right)^{2 H}\right)\left(\left(\frac{j+1}{N}\right)^{2 H}-\left(\frac{j}{N}\right)^{2 H}\right) E\left(B_{\frac{k+1}{N}}^{H}-B_{\frac{k}{N}}^{H}\right)\left(B_{\frac{j+1}{N}}^{H}-B_{\frac{j}{N}}^{H}\right) \\
=\frac{\sigma^{6}}{N} \sum_{\substack{k=0 \\
k=j,|k-j|=1}}^{N-1} \sum_{j=0}^{N-1}\left(2 H(k+1)^{2 H-1}\right)\left(2 H(j+1)^{2 H-1}\right) \times\left[\frac{1}{N^{2 H}}\right] \\
+\frac{\sigma^{6}}{N} \sum_{k=0}^{N-1} \sum_{j=0}^{N-1}\left(2 H(k+1)^{2 H-1}\right)\left(2 H(j+1)^{2 H-1}\right) \\
\quad \times \frac{1}{2}\left[\left|\frac{k-j+1}{N}\right|^{2 H}+\left|\frac{k-j-1}{N}\right|^{2 H}-2\left|\frac{k-j}{N}\right|^{2 H}\right]
\end{gathered}
$$




$$
\begin{aligned}
& \leq \frac{\sigma^{6}}{N^{2 H+1}} 4 H^{2}\left[N^{4 H-1}+2 N^{4 H-1}\right]+\frac{\sigma^{6}}{N} \sum_{\substack{k=0 \\
|k-j|>1}}^{N-1} \sum_{j=0}^{N-1}\left(2 H(k+1)^{2 H-1}\right)\left(2 H(j+1)^{2 H-1}\right) \\
& \quad \times\left[2 \frac{1}{N^{2}} 2 H(2 H-1)\left|\frac{k-j}{N}\right|^{2 H-2}\right] \\
& \leq 12 \sigma^{6} H^{2}\left[N^{2 H-2}\right]+\frac{16 \sigma^{6} H^{3}(2 H-1)}{N^{2 H+1}} \sum_{k=0}^{N-1} \sum_{j=0}^{N-1}(k+1)^{2 H-1}(j+1)^{2 H-1}|k-j|^{2 H-2} \\
& |k-j|>1 \\
& =12 \sigma^{6} H^{2}\left[N^{2 H-2}\right]+\frac{16 \sigma^{6} H^{3}(2 H-1)}{N^{2 H+1}} T_{1}
\end{aligned}
$$

Now,

$$
\begin{aligned}
T_{1} & =\sum_{k=1}^{N-1} k^{2 H-2} \sum_{j=1}^{N-k}\left(2 H j^{2 H-1}\right)\left(2 H(k+j)^{2 H-1}\right) \\
& \leq \sum_{k=1}^{N-1} k^{2 H-2} \sum_{j=1}^{N-k}\left(2 H(k+j)^{2 H-1}\right)^{2} \\
& =\sum_{k=1}^{N-1} k^{2 H-2} \sum_{j=k+1}^{N}\left(2 H j^{2 H-1}\right)^{2} \\
& \leq \sum_{k=1}^{N-1} k^{2 H-2} \sum_{j=1}^{N}\left(2 H j^{2 H-1}\right)^{2} \\
& \leq \sum_{k=1}^{N-1} k^{2 H-2}\left(4 H^{2} N^{4 H-1}\right) \\
& \leq N^{2 H-1} 4 H^{2} N^{4 H-1} \\
& =4 H^{2} N^{6 H-2}
\end{aligned}
$$

So, we get $E\left(U_{1}^{2}\right)<12 \sigma^{6} H^{2} N^{2 H-2}+16 \sigma^{6} H^{3}(2 H-1) N^{4 H-3} \rightarrow 0$ as $N \rightarrow \infty$ if $H<\frac{3}{4}$. Hence the result.

Lemma 5.2. Under the assumptions of theorem 5.1,

$$
\frac{\sigma^{4}}{4 \sqrt{N} h^{2 H}} \sum_{k=0}^{N-1}\left(\{(k+1) h\}^{2 H}-\{k h\}^{2 H}\right)^{2} \rightarrow 0
$$

Proof. Again putting $h=\frac{1}{N}$ l.h.s of (5.9) is $U_{2}=\frac{\sigma^{4}}{4} N^{2 H-\frac{1}{2}} \sum_{k=0}^{N-1}\left(\left|\frac{k+1}{N}\right|^{2 H}-\left|\frac{k}{N}\right|^{2 H}\right)^{2}$

$$
U_{2} \leq \frac{\sigma^{4}}{4} N^{-2 H-\frac{1}{2}} \sum_{k=0}^{N-1} 4 H^{2}\left[(k+1)^{2 H-1}\right]^{2} \leq \frac{\sigma^{4}}{4} N^{-2 H-\frac{1}{2}} 4 H^{2} N^{4 H-1}=\sigma^{4} H^{2} N^{2 H-\frac{3}{2}}
$$

So, $U_{2} \rightarrow 0$ as $n \rightarrow \infty$ if $2 H-\frac{3}{2}<0$ that is $H<\frac{3}{4}$. Hence the result.

We note that $\sigma_{H, 2}^{2}=2 \sigma^{4} \lim _{N \rightarrow \infty}\left(1+2\left(1-\frac{1}{N}\right)\left(2^{2 H}-1\right)^{2}+\sum_{k=2}^{N}\left(1-\frac{k}{N}\right)\left[(k+1)^{2 H}+(k-1)^{2 H}-2 k^{2 H}\right]^{2}\right)$. 
TABLE 1. The MEAN, VAR, MSE, ASYV of the estimators when $\sigma^{2}=0.4$

\begin{tabular}{|c|c|c|c|}
\hline \multicolumn{4}{|c|}{$\sigma^{2}=0.4$} \\
\hline H & 0.55 & 0.65 & 0.74 \\
MEAN & 0.399936 & 0.3986239 & 0.4016842 \\
VAR & 0.0002994642 & 0.00044069611 & 0.0007573279 \\
MSE & 0.0002994683 & 0.0004088546 & 0.0007601643 \\
\hline
\end{tabular}

TABle 2. The MEAN, VAR, MSE, ASYV of the estimators when $\sigma^{2}=1.6$

\begin{tabular}{|c|c|c|c|}
\hline \multicolumn{4}{|c|}{$\sigma^{2}=1.6$} \\
\hline H & 0.55 & 0.65 & 0.74 \\
MEAN & 1.601847 & 1.605567 & 1.607953 \\
VAR & 0.00456971 & 0.008053313 & 0.01498881 \\
MSE & 0.004573123 & 0.008084309 & 0.01505207 \\
\hline
\end{tabular}

TABle 3. The MEAN, VAR, MSE, ASYV of the estimators when $\sigma^{2}=6.4$

\begin{tabular}{|c|c|c|c|}
\hline \multicolumn{4}{|c|}{$\sigma^{2}=6.4$} \\
\hline H & 0.55 & 0.65 & 0.74 \\
MEAN & 6.401082 & 6.450209 & 6.720847 \\
VAR & 0.08589091 & 0.1253994 & 0.4416412 \\
MSE & 0.08589208 & 0.1279204 & 0.5445839 \\
\hline
\end{tabular}

5.2. Simulation Studies for fixed $\sigma$. In this section we present the simulation result for FBM driven model and it's estimator. We use somebm packages from $\mathrm{R}$ to simulate fractional Brownian motion. We keep drift parameter $\mu=0$. We generate each sample paths with $N=1000$ points and replicate with replication number, say $r=, 200$ times to find the mean, variance and mean squared error. We repeat the simulation for different values of $\sigma^{2}$ and for $H \in\left(\frac{1}{2}, \frac{3}{4}\right)$. Simulation shows that estimators are excellent when $H>\frac{1}{2}$ and $H<\frac{3}{4}$ for high frequency data with 1000 values in the time interval 0 to 1 .

5.3. Time varying $\sigma_{t}$. In this section we will prove the result for long memory process only, i.e. $H>\frac{1}{2}$. Our parameter of interest would be $\theta=\int_{\mathbb{R}}\left(M\left(\sigma_{s} \chi_{[0,1]}\right)\right)^{2} d s$.

To get the properties of time varying volatility estimator we need some Mathematical foundation. Readers are referred to Appendix for background for time varying volatility estimator before starting of this section. We denote

$$
\eta_{k}:=\int_{\mathbb{R}} \sigma_{s} \chi_{\left[\frac{k}{N}, \frac{k+1}{N}\right]} d B_{s}^{H}=\int_{\mathbb{R}} f_{k}(s) d B_{s}^{H}=I_{1}\left(f_{k}\right)
$$

where $f_{k}(s)=\sigma_{s} \chi_{\left[\frac{k}{N}, \frac{k+1}{N}\right]}(s)$ and $I_{1}$ is Wiener integral with respect to $\mathrm{fBm} B_{t}^{H}$ so $\eta_{k}$ is same as the Wiener integral discussed before. Define

$$
X_{N}=\sqrt{N}\left(\sum_{k=0}^{N-1} h^{-2 H+1} \eta_{k}^{2}-\tilde{\theta}_{N}\right)
$$

where $\tilde{\theta}_{N}=\sum_{k=0}^{N-1} h^{-2 H+1} E\left(\eta_{k}^{2}\right)$.

Theorem 5.2. Assume that the stock price follows the diffusion model specified by equation (4.4) with no drift $(\mu=0)$ and time varying volatility $\sigma_{t}$. Also assume that $H \in(1 / 2,3 / 4) . N \rightarrow \infty$ 
with the observation interval $N h=T$ remaining constant. Without loss of generality we can assume $T=1$. Then,

$$
X_{N} \Longrightarrow \mathcal{N}\left(0, \sigma_{H, 2, *}^{2}\right)
$$

where $\sigma_{H, 2, *}^{2}$ can be computed explicitly given the form of $\sigma(t)$ with the formula

$$
\sigma_{H, 2, *}^{2}=\lim _{N \rightarrow \infty} N^{4 H-1} 2 \sum_{k=0}^{N-1} \sum_{k^{\prime}=0}^{N-1}\left(\int_{\mathbb{R}} M f_{k}(s) M f_{k^{\prime}}(s) d s\right)^{2}
$$

Proof. r.h.s. of 5.10 can be rewritten as $X_{N}=\sqrt{N}\left(N^{2 H-1} \sum_{k=0}^{N-1} \eta_{k}^{2}-\tilde{\theta}_{N}\right)$.

Let us introduce some notations.

Let us denote $E\left(\eta_{k}^{2}\right)=\theta_{k}$. We get $\theta_{k}=\int_{\mathbb{R}}\left(M\left(\sigma_{s} \chi_{\left[\frac{k}{N}, \frac{k+1}{N}\right]}\right)\right)^{2} d s$ as expectation of Wiener integral $\int_{\mathbb{R}} \sigma_{s} \chi_{\left[\frac{k}{N}, \frac{k+1}{N}\right]} d B_{s}^{H}$. Now

$$
\begin{gathered}
E\left(N^{2 H-1} \sum_{k=0}^{N-1} \eta_{k}^{2}\right)=N^{2 H-1} \sum_{k=0}^{N-1} \theta_{k}=N^{2 H-1} \sum_{k=0}^{N-1} \int_{\mathbb{R}}\left(M\left(\sigma_{s} \chi_{\left[\frac{k}{N}, \frac{k+1}{N}\right]}\right)\right)^{2} d s \\
N^{2 H-1} \sum_{k=0}^{N-1} \int_{\mathbb{R}}\left(M\left(\sigma_{s} \chi_{\left[\frac{k}{N}, \frac{k+1}{N}\right]}\right)\right)^{2} d s=\tilde{\theta}_{N}
\end{gathered}
$$

If $H>1 / 2$ and $\sigma(s)<\Sigma \forall s$ then

$$
\begin{aligned}
\int_{\mathbb{R}} M f_{k}(s) M f_{j}(s) d s & \leq \Sigma^{2} H(2 H-1) \frac{|k-j|^{2 H-2}}{N^{2 H}} \text { for }|k-j|>1 \\
& \leq \Sigma^{2} \frac{1}{2 N^{2 H}} c \text { for }|k-j|=1, c=\left(2^{2 H}-1\right) \text { a constant } \\
& \leq \frac{\Sigma^{2}}{N^{2 H}} \text { for } k=j
\end{aligned}
$$

Using product formula for Wiener chaos integrals (8.7) we get

$$
\begin{aligned}
\eta_{k}^{2}= & I_{1}^{2}\left(f_{k}(s)\right) \\
= & I_{2}\left(f_{k} \otimes_{0} f_{k}\right)+I_{0}\left(f_{k} \otimes_{1} f_{k}\right) \\
= & I_{2}\left(f_{k} \otimes_{0} f_{k}\right)+\int_{\mathbb{R}}\left(M\left(\sigma_{s} \chi_{\left[\frac{k}{N}, \frac{k+1}{N}\right]}\right)\right)^{2} d s \\
& \quad \text { and } \eta_{k}^{2}-\theta_{k}=I_{2}\left(f_{k} \otimes_{0} f_{k}\right)
\end{aligned}
$$

We note that $I_{0}\left(f_{k} \otimes_{1} f_{k}\right)=\left\langle f_{k}, f_{k}\right\rangle_{\mathcal{H}}=\int_{\mathbb{R}}\left(M\left(\sigma_{s} \chi_{\left[\frac{k}{N}, \frac{k+1}{N}\right]}\right)\right)^{2} d s$.

Then $E\left(\eta_{k}^{2}\right)=E I_{2}\left(f_{k} \otimes_{0} f_{k}\right)+\int_{\mathbb{R}}\left(M\left(\sigma_{s} \chi_{\left[\frac{k}{N}, \frac{k+1}{N}\right]}\right)\right)^{2} d s$. So, we get $E I_{2}\left(f_{k} \otimes_{0} f_{k}\right)=0$.

Let us now calculate the second moment of $\sum_{k=0}^{N-1} \eta_{k}^{2}$. 


$$
\begin{aligned}
\mathrm{E}\left(\sum_{k=0}^{N-1} \eta_{k}^{2}\right)^{2}= & \sum_{k=0}^{N-1} \sum_{k^{\prime}=0}^{N-1} \mathrm{E}\left(\left(I_{2}\left(f_{k} \otimes_{0} f_{k}\right)+\theta_{k}\right)\left(I_{2}\left(f_{k^{\prime}} \otimes_{0} f_{k^{\prime}}\right)+\theta_{k}\right)\right) \\
= & \sum_{k=0}^{N-1} \sum_{k^{\prime}=0}^{N-1}\left[\mathrm{E}\left(I_{2}\left(f_{k} \otimes_{0} f_{k}\right) I_{2}\left(f_{k^{\prime}} \otimes_{0} f_{k^{\prime}}\right)\right)\right. \\
& \left.+\theta_{k} \mathrm{E} I_{2}\left(f_{k^{\prime}} \otimes_{0} f_{k^{\prime}}\right)+\theta_{k}^{\prime} \mathrm{E} I_{2}\left(f_{k} \otimes_{0} f_{k}\right)+\theta_{k} \theta_{k}^{\prime}\right] \\
= & A_{1}+A_{2}+A_{3}+A_{4}
\end{aligned}
$$

Where $A_{1}, A_{2}, A_{3}, A_{4}$ are respective terms in the summation. Now $A_{2}=A_{3}=0$. We observe that $\operatorname{Var}\left(\sum_{k=0}^{N-1} \eta_{k}^{2}\right)=A_{1}$

$$
\begin{aligned}
A_{1} & =2 \sum_{k=0}^{N-1} \sum_{k^{\prime}=0}^{N-1}\left\langle f_{k} \otimes_{0} f_{k}, f_{k^{\prime}} \otimes_{0} f_{k^{\prime}}\right\rangle_{\mathcal{H}^{\otimes 2}} \\
& =2 \sum_{k=0}^{N-1} \sum_{k^{\prime}=0}^{N-1}\left\langle f_{k}, f_{k^{\prime}}\right\rangle_{\mathcal{H}^{\otimes 1}}^{2} \\
& =2 \sum_{k=0}^{N-1} \sum_{k^{\prime}=0}^{N-1}\left(\mathrm{E}\left(I_{1}\left(f_{k}\right) I_{1}\left(f_{k^{\prime}}\right)\right)^{2}\right. \\
& =2 \sum_{k=0}^{N-1} \sum_{k^{\prime}=0}^{N-1}\left(\mathrm{E}\left(\eta_{k} \eta_{k^{\prime}}\right)\right)^{2} \\
& =2 \sum_{k=0}^{N-1} \sum_{k^{\prime}=0}^{N-1}\left(\int_{\mathbb{R}} M f_{k}(s) M f_{k^{\prime}}(s) d s\right)^{2}
\end{aligned}
$$

$$
\begin{aligned}
A_{1} & \approx 2\left(N^{1-4 H}+\sum_{k=0}^{N-1} \sum_{\substack{k^{\prime}=0 \\
k \neq k^{\prime}}}^{N-1}\left(\Sigma^{2} H(2 H-1)\left|\frac{k^{\prime}-k}{N}\right|^{2 H-2} \frac{1}{N^{2}}\right)^{2}\right) \\
& =2\left(N^{1-4 H}+\Sigma^{4} H^{2}(2 H-1)^{2} N^{-4 H} \sum_{\substack { k=0 \\
\begin{subarray}{c}{k^{\prime}=0 \\
k \neq k^{\prime}{ k = 0 \\
\begin{subarray} { c } { k ^ { \prime } = 0 \\
k \neq k ^ { \prime } } }\end{subarray}}^{N-1}\left|k^{\prime}-k\right|^{4 H-4}\right) \\
& =2\left(N^{1-4 H}+\Sigma^{4} H^{2}(2 H-1)^{2} N^{-4 H} \sum_{\substack{k=-N+1 \\
k \neq 0}}^{N-1} \sum_{k^{\prime}=k}^{N-1+k}|k|^{4 H-4}\right) \\
& =2 N^{1-4 H}+4 \Sigma^{4} H^{2}(2 H-1)^{2} N^{-4 H}(N-1) \sum_{k=1}^{N-1} k^{4 H-4} \text { for } H>1 / 2
\end{aligned}
$$

Then $N^{4 H-1} A_{1}<\infty$ if $H<\frac{3}{4}$. So, we can see $N^{4 H-1} E\left(\sum_{k=0}^{N-1} \eta_{k}^{2}\right)^{2}=N^{4 H-1} A_{1}$. 
Let us write $X_{N}$ in terms of multiple Wiener Ito integral.

$$
\begin{aligned}
X_{N} & =\sqrt{N}\left(N^{2 H-1} \sum_{k=0}^{N-1}\left(I_{1}\left(f_{k}\right)\right)^{2}-\tilde{\theta}_{N}\right) \\
& =\sqrt{N}\left(N^{2 H-1} \sum_{k=0}^{N-1}\left(I_{2}\left(f_{k} \otimes_{0} f_{k}\right)+\theta_{k}\right)-\tilde{\theta}_{N}\right) \\
& =N^{2 H-\frac{1}{2}} I_{2}\left(\sum_{k=0}^{N-1}\left(f_{k} \otimes_{0} f_{k}\right)\right)+\sqrt{N}\left(N^{2 H-1} \sum_{k=0}^{N-1} \theta_{k}-\tilde{\theta}_{N}\right) \\
& =Y_{N}+\sqrt{N}\left(N^{2 H-1} \sum_{k=0}^{N-1} \theta_{k}-\tilde{\theta}_{N}\right)
\end{aligned}
$$

Now $\sqrt{N}\left(N^{2 H-1} \sum_{k=0}^{N-1} \theta_{k}-\tilde{\theta}_{N}\right)=0$ for all $N$. Observe that $E\left(Y_{N}^{2}\right)=N^{4 H-1} A_{1}=S_{N}$. Define $G_{N}=\frac{Y_{N}}{\sqrt{S_{N}}}$

To prove asymptotic normality we will use the two theorems (8.1) and (8.2). Using the theorems we want to show that $\left\|D G_{N}\right\|_{\mathcal{H}}^{2} \rightarrow 2$ in $L^{2}$. For that matter we first show $\lim _{N \rightarrow \infty} E\left[\left\|D G_{N}\right\|_{\mathcal{H}}^{2}\right]=2$ and then $\lim _{N \rightarrow \infty} E\left[\left\|D G_{N}\right\|_{\mathcal{H}}^{2}-2\right]^{2}=0$. Now,

$$
\begin{aligned}
& {\left[\left\|D G_{N}\right\|_{\mathcal{H}}^{2}-2\right]^{2} } \\
= & {\left[\left\|D G_{N}\right\|_{\mathcal{H}}^{2}-E\left[\left\|D G_{N}\right\|_{\mathcal{H}}^{2}\right]+E\left[\left\|D G_{N}\right\|_{\mathcal{H}}^{2}\right]-2\right]^{2} } \\
= & {\left[\left\|D G_{N}\right\|_{\mathcal{H}}^{2}-E\left[\left\|D G_{N}\right\|_{\mathcal{H}}^{2}\right]^{2}+\left[E\left[\left\|D G_{N}\right\|_{\mathcal{H}}^{2}\right]-2\right]^{2}+2\left[\left\|D G_{N}\right\|_{\mathcal{H}}^{2}-E\left[\left\|D G_{N}\right\|_{\mathcal{H}}^{2}\right]\left[E\left[\left\|D G_{N}\right\|_{\mathcal{H}}^{2}\right]-2\right]\right.\right.} \\
= & A+B+2 C
\end{aligned}
$$

where $A, B, C$ are respective terms. Now $E C=0, B \rightarrow 0$ as $N \rightarrow \infty$. We will be interested in $A$ for further analysis.

Using 8.11) we get

$$
D_{t} Y_{N}=2 N^{2 H-\frac{1}{2}} \sum_{j=0}^{N-1} f_{j}(t) I_{1}\left(f_{j}\right)
$$

So

$$
\left\|D Y_{N}\right\|_{\mathcal{H}}^{2}=4 N^{4 H-1} \sum_{k=0}^{N-1} \sum_{j=0}^{N-1} I_{1}\left(f_{j}\right) I_{1}\left(f_{k}\right)\left\langle f_{j}, f_{k}\right\rangle_{\mathcal{H}}
$$

Now

$$
E\left[\left\|D Y_{N}\right\|_{\mathcal{H}}^{2}\right]=4 N^{4 H-1} \sum_{k=0}^{N-1} \sum_{j=0}^{N-1}\left(\left\langle f_{j}, f_{k}\right\rangle_{\mathcal{H}}\right)^{2}=4 N^{4 H-1} \frac{A_{1}}{2}=2 S_{N}
$$

We note that $E\left[\left\|D Y_{N}\right\|_{\mathcal{H}}^{2}\right]=2 E\left[Y_{N}^{2}\right]$. So $E\left[\left\|D G_{N}\right\|_{\mathcal{H}}^{2}\right] \rightarrow 2$ as $N \rightarrow \infty$. Let us calculate the following

$$
\begin{aligned}
& \left\|D Y_{N}\right\|_{\mathcal{H}}^{2}-E\left[\left\|D Y_{N}\right\|_{\mathcal{H}}^{2}\right] \\
= & 4 N^{4 H-1} \sum_{k=0}^{N-1} \sum_{j=0}^{N-1}\left[\left(I_{2}\left(f_{j} \otimes_{0} f_{k}\right)+I_{0}\left(f_{k} \otimes_{1} f_{k}\right)\left\langle f_{j}, f_{k}\right\rangle_{\mathcal{H}}-\left\langle f_{j}, f_{k}\right\rangle_{\mathcal{H}}^{2}\right]\right. \\
= & 4 N^{4 H-1} \sum_{k=0}^{N-1} \sum_{j=0}^{N-1}\left[I_{2}\left(f_{j} \otimes_{0} f_{k}\right)\left\langle f_{j}, f_{k}\right\rangle_{\mathcal{H}}\right]
\end{aligned}
$$


And then

$$
\begin{aligned}
E\left[\left\|D Y_{N}\right\|_{\mathcal{H}}^{2}-E\left[\left\|D Y_{N}\right\|_{\mathcal{H}}^{2}\right]\right]^{2} & =16 N^{8 H-2} E\left[\sum_{k=0}^{N-1} \sum_{j=0}^{N-1}\left[I_{2}\left(f_{j} \otimes_{0} f_{k}\right)\left\langle f_{j}, f_{k}\right\rangle_{\mathcal{H}}\right]\right]^{2} \\
& \leq \text { constant } N^{8 H-6} \rightarrow 0
\end{aligned}
$$

For last part of the calculation see lemma 5.2 of Tudor (2013). as $N \rightarrow \infty$ and $H<\frac{3}{4}$. Hence the proof.

Theorem 5.3. Under the conditions of theorem 5.2,

$$
\sqrt{N}\left(\hat{\sigma}^{2}-\tilde{\theta}_{N}\right) \Longrightarrow \mathcal{N}\left(0, \sigma_{H, 2, *}^{2}\right)
$$

where $\sigma_{H, 2, *}^{2}$ is a constant that can be computed explicitly, given the form of $\sigma(t)$.

Proof. Under the condition of $\mu=0$, the solution (3.1) of the stochastic differential equation (4.2) simplifies to

$$
S_{t}=S_{0} \exp \left(\int_{0}^{t} \sigma_{s} d B_{s}^{H}-\frac{1}{2} \int_{\mathbb{R}}\left(M\left(\sigma_{s} \chi_{[0, t]}\right)\right)^{2} d s\right)
$$

Let us denote $\tilde{f}_{k}(s)=\sum_{j=0}^{k} f_{k}(s)$ and $\delta_{k}=\int_{\mathbb{R}}\left(M \tilde{f}_{k+1}(s)\right)^{2}-\int_{\mathbb{R}}\left(M \tilde{f}_{k}(s)\right)^{2}$. Putting the solution (5.13) in the definition of $\hat{\sigma^{2}}$ in equation (5.1), we get,

$$
\begin{aligned}
\hat{\sigma^{2}} & =\frac{1}{N h^{2 H}} \sum_{k=0}^{N-1}\left[\eta_{k}-\frac{1}{2} \delta_{k}\right]^{2} \\
& =N^{2 H-1}\left[\sum_{k=0}^{N-1} \eta_{k}^{2}-\sum_{k=0}^{N-1} \eta_{k} \delta_{k}+\frac{1}{4} \sum_{k=0}^{N-1} \delta_{k}^{2}\right]
\end{aligned}
$$

Combining (5.14) and (5.11), we have

$$
\sqrt{N}\left(\hat{\sigma^{2}}-\tilde{\theta}_{N}\right)=X_{N}-N^{2 H-\frac{1}{2}} \sum_{k=0}^{N-1} \eta_{k} \delta_{k}+\frac{1}{4} N^{2 H-\frac{1}{2}} \sum_{k=0}^{N-1} \delta_{k}^{2}
$$

where $X_{N}$ is defined in theorem 5.2. It is shown in lemma 5.3 that the second term converges to zero in $L^{2}$ as $N \rightarrow \infty$. In lemma 5.4 it is shown that the third term converges to zero. The theorem now follows by applying Chebyshev and Slutsky with theorem 5.2 as before.

Lemma 5.3. Under the assumptions of theorem 5.2,

$$
G=N^{2 H-\frac{1}{2}} \sum_{k=0}^{N-1} \eta_{k} \delta_{k} \stackrel{L^{2}}{\longrightarrow} 0
$$

Proof. Let us recall

$$
\begin{gathered}
\mathrm{E} \eta_{k} \eta_{k^{\prime}}=\int_{\mathbb{R}} M f_{k}(s) M f_{k^{\prime}}(s) d s \\
\delta_{k}=\int_{\mathbb{R}}\left[\left(M \tilde{f}_{k+1}(s)\right)^{2}-\left(M \tilde{f}_{k}(s)\right)^{2}\right] d s
\end{gathered}
$$


For $H>\frac{1}{2}$

$$
\begin{aligned}
\delta_{k} & =H(2 H-1)\left[\int_{0}^{\frac{k+1}{N}} \int_{0}^{\frac{k+1}{N}} \sigma(s) \sigma(t)|s-t|^{2 H-2} d s d t-\int_{0}^{\frac{k}{N}} \int_{0}^{\frac{k}{N}} \sigma(s) \sigma(t)|s-t|^{2 H-2} d s d t\right] \\
& =H(2 H-1)\left[\int_{\frac{k}{N}}^{\frac{k+1}{N}} \int_{\frac{k}{N}}^{\frac{k+1}{N}}+2 \int_{0}^{\frac{k}{N}} \int_{\frac{k}{N}}^{\frac{k+1}{N}}\right]
\end{aligned}
$$

So

$$
\begin{aligned}
\left|\delta_{k}\right| & \leq \frac{\Sigma^{2}}{N^{2 H}}\left[1+(k+1)^{2 H}-(k)^{2 H}-1\right] \\
& \approx \frac{\Sigma^{2}}{N^{2 H}}(k+1)^{2 H-1}
\end{aligned}
$$

We look at the $L_{2}$ norm of $G$.

$$
\begin{aligned}
E\left(G^{2}\right) & =N^{4 H-1} \sum_{k=0}^{N-1} \sum_{k^{\prime}=0}^{N-1} \delta_{k} \delta_{k^{\prime}} \int_{\mathbb{R}} M f_{k}(s) M f_{k^{\prime}}(s) d s \\
& \rightarrow 0 .
\end{aligned}
$$

using estimates for $\delta_{k}$ and estimates of $\int_{\mathbb{R}} M f_{k}(s) M f_{k^{\prime}}(s) d s$.

Lemma 5.4. Under the assumptions of theorem 5.2, then

$$
N^{2 H-\frac{1}{2}} \sum_{k=0}^{N-1} \delta_{k}^{2} \rightarrow 0
$$

Proof. Again using the estimates of $\delta_{k}$ we have

$$
\begin{aligned}
N^{2 H-\frac{1}{2}} \sum_{k=0}^{N-1} \delta_{k}^{2} & \leq N^{2 H-\frac{1}{2}} \sum_{k=0}^{N-1}\left(\frac{\Sigma^{2}}{N^{2 H}}(k+1)^{2 H-1}\right)^{2} \\
& =\Sigma^{4} N^{-2 H-\frac{1}{2}} N^{4 H-1}
\end{aligned}
$$

This proves the lemma for $H>\frac{1}{2}$ as well as $H<\frac{3}{4}$.

5.4. Simulation studies (time varying volatility). In this section we did simulation studies to see the difference between our actual parameter of interest $\int_{\mathbb{R}}\left(M\left(\sigma(s) \chi_{[0,1]}\right)\right)^{2} d s$ and what we achieve $\tilde{\theta}_{N}$ for sample size $N$, for different Hurst parameter with different $\sigma(s)$ function. We have chosen different functions $\sigma(t)$, necessarily bounded, on the interval [0,1], calculate $\tilde{\theta}_{N}$ and report the results. We take $N=1000$ and compute $\tilde{\theta}_{N}$ and $\theta$ and note the difference.

Let us consider $\sigma(t)$ a sub linear function of the form $\sigma(t)=\sigma t^{\alpha}, t \in(0,1), \frac{1}{2}<H<\frac{3}{4}$, $\sigma>0,0<\alpha<1$. Next we consider functions of the form $\sigma(t)=\sigma\left(t^{\alpha}+t^{\beta}\right)$ for $t \in(0,1), 0<\alpha<1$ and $\beta>1$, i.e. polynomial with positive fraction and integer powers. We note that $\tilde{\theta}_{N}$ may not converge as $N \rightarrow \infty$ and it will indeed not converges. So the simulation result is only for $N=1000$.

For practical purpose the sub linear functions seems best.

\section{Conclusions}

In this paper we sketch the way to obtain the option price for fBm driven model with time varying volatility. We identify the parameter of interest for calculating option price. Next we have proposed estimator from high frequency data for parameter similar to so called "integrated volatility", in case of constant volatility and time varying volatility model driven by $\mathrm{fBm}$. We have shown that estimators are asymptotically normally distributed for $H<\frac{3}{4}$. For time varying volatility model, the 
TABLE 4. The comparison of $\tilde{\theta}_{N}$ and $\theta$

\begin{tabular}{|c|c|c|c|}
\hline \multicolumn{4}{|c|}{$\sigma=0.4, \alpha=0.3$} \\
\hline $\mathrm{H}$ & 0.55 & 0.65 & 0.74 \\
$\tilde{\theta}_{N}$ & 0.1000039 & 0.1000005 & 0.09992656 \\
$\theta$ & 0.09962605 & 0.09868432 & 0.09770835 \\
\hline \multicolumn{4}{|c|}{$\sigma=6.4, \alpha=0.3$} \\
\hline $\mathrm{H}$ & 0.55 & 0.65 & 0.74 \\
$\tilde{\theta}_{N}$ & 25.60027 & 25.60061 & 25.6002 \\
$\theta$ & 25.50445 & 25.2632 & 25.01328 \\
\hline
\end{tabular}

TABLE 5. The comparison of $\tilde{\theta}_{N}$ and $\theta$

\begin{tabular}{|c|c|c|c|}
\hline \multicolumn{4}{|c|}{$\sigma=0.4, \alpha=0.8, \beta=2$} \\
\hline $\mathrm{H}$ & 0.55 & 0.65 & 0.74 \\
$\tilde{\theta}_{N}$ & 0.1777476 & 0.1777324 & 0.1776835 \\
$\theta$ & 0.1705813 & 0.1579971 & 0.1482963 \\
\hline \multicolumn{4}{|c|}{$\sigma=6.4, \alpha=0.8, \beta=2$} \\
\hline $\mathrm{H}$ & 0.55 & 0.65 & 0.74 \\
$\tilde{\theta}_{N}$ & 45.50396 & 45.50438 & 45.5041 \\
$\theta$ & 43.66903 & 40.44747 & 37.96394 \\
\hline
\end{tabular}

estimator will not asymptotically unbiased for our parameter of interest. Through some simulation study we showed how close of the parameter of interest can be achieved by the estimators under consideration.

\subsection{Future directions.}

(1) In all these we assume $H$ as a known quantity. The estimation for $H$ also exists separately. See Prakasa Rao (2010), Breton et al. (2009). Is there any way to combine?

(2) Following Zhang et al (2005) we want to develop method of inferring volatility when the process is observed discretely with noise.

(3) Following Barndorff-Nielsen and Shephard (2004) we want to consider estimators for jump process.

\subsection{Comments.}

- Why consider fBM driven models? Non-stationary time series will also take care of thick-tails and long-range dependence in returns. But it is not easy to put them in an option pricing framework.

- Why do we have confidence intervals for option prices, when looked at as solution of an optimization problem? There is uncertainty in utility/preferences.

\section{ACKNOWLEDGEMENT}

First author wants to acknowledge Department of Science and Technology, India, for financial support to conduct this research work.

\section{ApPENDix}

8.1. Wiener integral . $\Omega:=\mathcal{S}^{\prime}(\mathbb{R})$, dual of the space of Schwartz class functions $\mathcal{S}$, is tempered distributions with sigma algebra $\mathcal{F}$. $\langle\omega, f\rangle$ is the random variable by action of $\omega \in \Omega$ on $f \in \mathcal{S}(\mathbb{R})$. Bochnor Minlos probability measure $P$ on $(\Omega, \mathcal{F})$ is such that $E[\exp i\langle\omega, f\rangle]=e^{-\frac{1}{2}\|f\|^{2}},\|f\|^{2}=$ 
$\int_{\mathbb{R}} f^{2}(x) d x$. We also have expectation $E[\langle\omega, f\rangle]=0$ and variance $E[\langle\omega, f\rangle]^{2}=\|f\|^{2}$ under $P$. Let $I(0, t)$ is indicator function. Then $\tilde{B}_{t}(\omega)=\langle\omega, I(0, t)\rangle \in L^{2}(P)$ and $\tilde{B}_{t}$ is Gaussian random variable for each $t$. Using Kolmogorov's continuity theorem $\tilde{B}_{t}$ has continuous version as $B_{t}$ and $B_{t}$ is standard Brownian motion. This duality can be extended for $f \in L^{2}(\mathbb{R})$ approximating $f$ by step functions we get $\langle\omega, f\rangle=\int_{\mathbb{R}} f(t) d B_{t}(\omega)$.

Let $M(0, t)=M I(0, t) \in L^{2}(\mathbb{R})$. Then $\tilde{B}_{t}^{H}(\omega)=\langle\omega, M(0, t)\rangle$. $\tilde{B}_{t}^{H}$ is Gaussian random variable with $E \tilde{B}_{t}^{H}=0$ and $E \tilde{B}_{t}^{H} \tilde{B}_{s}^{H}=\int_{\mathbb{R}} M(0, t)(x) M(0, s)(x) d x=\frac{1}{2}\left(t^{2 H}+s^{2 H}-|t-s|^{2 H}\right)$. Again take continuous version of $\tilde{B}_{t}^{H}$ as $B_{t}^{H}$. So we get $B_{t}^{H}=\int_{\mathbb{R}}\left(M I(0, t)(s) d B_{s}\right.$.

Let $f \in L_{H}^{2}(\mathbb{R})$, deterministic. Then $M f \in L^{2}(\mathbb{R})$. The Wiener integral with respect to fractional Brownian motion is defined as

$$
\int_{\mathbb{R}} f(s) d B_{s}^{H}=\int_{\mathbb{R}}(M f)(s) d B_{s}
$$

Take $f \in \mathcal{S}$. Construct Bochnor Minlos probability measure $P^{H}$ on $(\Omega, \mathcal{F})$ is such that $E\left[\exp i\langle\omega, f\rangle_{H}\right]=$ $e^{-\frac{1}{2}\|f\|_{H}^{2}},\|f\|_{H}^{2}=\|f\|_{L_{H}^{2}}=\|M f\|_{L^{2}}=\int_{\mathbb{R}}(M f)^{2}(x) d x$. We also have expectation $E^{H}\left[\langle\omega, f\rangle_{H}\right]=0$ and variance $E^{H}\left[\langle\omega, f\rangle_{H}\right]^{2}=\|f\|_{H}^{2}$ under $P^{H}$. Let $I(0, t)$ is indicator function. Then $\tilde{B}_{t}^{H}(\omega)=$ $\langle\omega, I(0, t)\rangle_{H} \in L^{2}\left(P^{H}\right)$ and $\tilde{B}_{t}^{H}$ is Gaussian random variable for each $t$. Using Kolmogorov's continuity theorem $\tilde{B}_{t}^{H}$ has continuous version as $B_{t}^{H}$ and $B_{t}^{H}$ is fractional Brownian motion. This duality can be extended for $f \in L_{H}^{2}(\mathbb{R})$ we get $\langle\omega, f\rangle_{H}=\int_{\mathbb{R}} f(t) d B_{t}^{H}(\omega)$. So, we note that the term $\int_{0}^{t} \sigma_{s} d B_{s}^{H}$ appears in solution of the SDE (3.1) is a Wiener integral with respect to Brownian motion. The first two moments of Wiener integral with respect to fractional Brownian motion are as follows

$$
E\left(\int_{\mathbb{R}} f(s) d B_{s}^{H}\right)=0
$$

and

$$
\begin{gathered}
E\left[\int_{\mathbb{R}} f(s) d B_{s}^{H}\right]^{2}=\|f\|_{L_{H}^{2}}=\|M f\|_{L^{2}} \\
E\left[\int_{\mathbb{R}} f(s) d B_{s}^{H} \int_{\mathbb{R}} g(s) d B_{s}^{H}\right]=\langle f, g\rangle_{L_{H}^{2}}
\end{gathered}
$$

8.2. Wick product and related topics . This section consists of background material required for section 4 .

Chaos expansion Theorem Let $F \in L^{2}(P)$. Then there exists a unique family $c_{\alpha}, \alpha \in \mathcal{I}$ of constants, $c_{\alpha} \in \mathbb{R}$ such that $F(\omega)=\sum_{\alpha \in \mathcal{I}} c_{\alpha} H_{\alpha}(\omega), H_{\alpha}$ is multi indexed Hermite polynomial of Bm (convergence in $\left.L^{2}(P)\right)$. Moreover, we have the isometry $E\left(F^{2}\right)=\sum_{\alpha \in \mathcal{I}} c_{\alpha}^{2} \alpha$ !.

Let us define $(\mathcal{S})$ as space of stochastic test functions and $\left(\mathcal{S}^{*}\right)$ as space of stochastic distributions.

$(\mathcal{S})$ is collection of all $F \in L^{2}(P)$ such that it's expansion is $F(\omega)=\sum_{\alpha \in \mathcal{I}} c_{\alpha} H_{\alpha}(\omega)$ where $\|F\|_{k}^{2}=$ $\sum_{\alpha} \alpha ! c_{\alpha}^{2}(2 \mathbb{N})^{k \alpha}<\infty$ for all integer $k=1,2, \cdots$ with $(2 \mathbb{N})^{k \gamma}=\prod_{j=1}^{m}(2 j)^{\gamma_{j}}, \gamma=\left(\gamma_{1}, \cdots, \gamma_{m}\right) \in \mathcal{I}$. 
$\left(\mathcal{S}^{*}\right)$ is collection of all $G \in L^{2}(P)$ such that it's expansion is $G(\omega)=\sum_{\beta \in \mathcal{I}} c_{\beta} H_{\beta}(\omega)$ where $\|G\|_{q}^{2}=$ $\sum_{\beta} \beta ! c_{\beta}^{2}(2 \mathbb{N})^{-q \beta}<\infty$ for some integer $q<\infty$.

$\left(\mathcal{S}^{*}\right)$ is dual of $(\mathcal{S})$ with duality relation as follows:

If $F(\omega)=\sum_{\alpha \in \mathcal{I}} a_{\alpha} H_{\alpha}(\omega) \in(\mathcal{S})$ and $G(\omega)=\sum_{\alpha \in \mathcal{I}} b_{\alpha} H_{\alpha}(\omega) \in\left(\mathcal{S}^{*}\right)$ then action of $G$ on $F$ is $\langle G, F\rangle_{\left(\mathcal{S}^{*}\right),(\mathcal{S})}=\sum_{\alpha \in \mathcal{I}} \alpha ! a_{\alpha} b_{\alpha}$

If $L^{2}(P) \subset\left(\mathcal{S}^{*}\right)$ and $(\mathcal{S}) \subset L^{2}(P)$ then action of $G$ on $F$ is $\langle G, F\rangle_{\left(\mathcal{S}^{*}\right),(\mathcal{S})}=\langle G, F\rangle_{L^{2}(P)}=E(G F)$. If $F(\omega)=\sum_{\alpha \in \mathcal{I}} a_{\alpha} H_{\alpha}(\omega) \in\left(\mathcal{S}^{*}\right)$ and $G(\omega)=\sum_{\beta \in \mathcal{I}} b_{\beta} H_{\beta}(\omega) \in\left(\mathcal{S}^{*}\right)$ then Wick product $\diamond$ is defined as $(F \diamond G)(\omega)=\sum_{\alpha, \beta \in \mathcal{I}} a_{\alpha} b_{\beta} H_{\alpha+\beta}(\omega) \in\left(\mathcal{S}^{*}\right)$.

Fractional white noise $W_{t}^{H}=\frac{d B_{t}^{H}}{d t}$ is an element of $\left(\mathcal{S}^{*}\right)$ see Elliott and Van der Hoek (2003), Biagini et al. (2004) for detail.

Wick Ito Skorohod integral with respect to $B_{t}^{H}:$ If $Y: \mathbb{R} \rightarrow\left(\mathcal{S}^{*}\right)$ is such that $Y_{t} \diamond W_{t}^{H}$ is integrable in $\left(\mathcal{S}^{*}\right)$ then we define

$$
\int_{\mathbb{R}} Y_{t} d B_{t}^{H}=\int_{\mathbb{R}} Y_{t} \diamond W_{t}^{H} d t
$$

If $f \in L_{H}^{2}(\mathbb{R})$ then

$$
\int_{\mathbb{R}} f(s) d B_{s}^{H}=\int_{\mathbb{R}} f(s) \diamond W_{s}^{H} d s=\int_{\mathbb{R}}(M f)(s) d B_{s} .
$$

8.3. Background to deal with time varying volatility estimator . In this section we introduce some notations and established results which will be needed for our future calculation. Our fractional Brownian motion $B_{t}^{H}$ is centered, continuous, mean zero Gaussian processes with covariance functions as $R_{B}^{H}=\operatorname{cov}\left(B_{s}^{H}, B_{t}^{H}\right)=\frac{1}{2}\left[t^{2 H}+s^{2 H}-|t-s|^{2 H}\right]$. Let $\mathcal{E}$ be the set of real valued step functions. For $\phi=I[0, t], \psi=I[0, s] \in \mathcal{E}$ let us define inner product $\langle\phi, \psi\rangle_{\mathcal{E}}=\left\langle I_{[0, s]}, I_{[0, t]}\right\rangle_{\mathcal{E}}=R_{B}^{H}$. For $\phi=\sum_{j} a_{j} I\left[0, t_{j}\right]$, set $B^{H}(\phi)=\sum_{j} a_{j} B_{t_{j}}^{H}$. Let $\psi=\sum_{j} b_{j} I\left[0, t_{j}\right]$. So, $E\left(B^{H}(\phi) B^{H}(\psi)\right)=\langle\phi, \psi\rangle_{\mathcal{E}}$. Next for $\phi \in \mathcal{H}$, there are $\phi_{n} \in \mathcal{E}$ such that $\phi_{n} \rightarrow \phi$ in $\mathcal{H}$ then $B^{H}(\phi)$ is the $L^{2}$ limit of $B^{H}\left(\phi_{n}\right)$. So we get $\langle\phi, \psi\rangle_{\mathcal{H}}=E B^{H}(\phi) B^{H}(\psi) .\left\{B^{H}(\phi), \phi \in \mathcal{H}\right\}$ is called isonormal Gaussian process.

Let $H_{n}$ be $n$th Hermite polynomial satisfying

$$
\frac{d}{d x} H_{n}(x)=H_{n-1}(x), n \geq 1 .
$$

Take $\phi \in \mathcal{H}$ such that $\|\phi\|_{\mathcal{H}}=1$. Consider random variables $H_{n}\left(B^{H}(\phi)\right)$ and take the closure of the span of these random variables. This is the $n$th order Wiener chaos $\mathcal{W}_{n}$.

$I_{n}$, the multiple stochastic (Wiener Ito) integral with respect to isonormal Gaussian process $B^{H}$, is a map from $\mathcal{H}^{\odot n}$ to $\mathcal{W}_{n}, \mathcal{H}^{\odot n}$ being symmetric tensor product of $\mathcal{H}$. $\mathcal{H}^{\odot n}$ has norm $\frac{1}{\sqrt{n !}}\|\cdot\|_{\mathcal{H} \otimes n}$, $\mathcal{H}^{\otimes n}$ is tensor product of $\mathcal{H}$.

Then for $f \in \mathcal{H}^{\odot n}$, we also have $I_{n}(f)=I_{n}(\tilde{f}), \tilde{f}$ is symmetrization of $f$.

For $\phi \in \mathcal{H}, I_{n}\left(\phi^{\otimes n}\right)=n ! H_{n}\left(I_{1}(\phi)\right)=n ! H_{n}\left(B^{H}(\phi)\right)$ is linear isometry between $\mathcal{H}^{\odot n}$ and $\mathcal{W}_{n}$.

Now for $f \in \mathcal{H}^{\odot n}$ and $g \in \mathcal{H}^{\odot m}$ we have followings:

$$
\begin{aligned}
& E\left(I_{n}(f) I_{m}(g)\right)=n !\langle\tilde{f}, \tilde{g}\rangle_{\mathcal{H} \otimes n} \text { if } m=n \\
& E\left(I_{n}(f) I_{m}(g)\right)=0 \text { if } m \neq n
\end{aligned}
$$


Let $\left\{e_{i}, i \geq 1\right\}$ be an orthonormal basis of $\mathcal{H}, m, n \geq 1, r=0, \cdots, n \wedge m . f \otimes_{r} g \in \mathcal{H}^{\otimes(m+n-2 r)}$ is contraction is defined as

$$
f \otimes_{r} g=\sum_{i 1, \cdots, i r=1}^{\infty}\left\langle f, e_{i 1} \otimes \cdots \otimes e_{i r}\right\rangle_{\mathcal{H}} \otimes r\left\langle g, e_{i 1} \otimes \cdots \otimes e_{i r}\right\rangle_{\mathcal{H}} \otimes r .
$$

This definition does not depend on the choice of orthonormal basis and $\left\langle f, e_{i 1} \otimes \cdots \otimes e_{i r}\right\rangle_{\mathcal{H} \otimes r} \in \mathcal{H}^{\odot(n-r)}$, $\left\langle g, e_{i 1} \otimes \cdots \otimes e_{i r}\right\rangle_{\mathcal{H}}^{\otimes r} \in \mathcal{H}^{\odot(m-r)} . f \otimes_{r} g$ is not necessarily symmetric. Let $f \tilde{\otimes}_{r} g$ is symmetrization of $f \otimes_{r} g$. Then

$$
I_{n}(f) I_{m}(g)=\sum_{r=0}^{m \wedge n} r !\left(\begin{array}{c}
n \\
r
\end{array}\right)\left(\begin{array}{c}
m \\
r
\end{array}\right) I_{n+m-2 r}\left(f \tilde{\otimes}_{r} g\right) .
$$

Also for $n=m=r$ we have

$$
I_{0}\left(f \otimes_{r} g\right)=\langle f, g\rangle_{\mathcal{H}}{ }^{\otimes r} .
$$

Let $F$ be a functional of the isonormal Gaussian process $B^{H}$ such that $E\left(F\left(B^{H}\right)^{2}\right)<\infty$ then there is unique sequence $f_{n} \in \mathcal{H}^{\odot n}$ and $F$ can be written as sum of multiple stochastic integrals as $F=\sum_{n \geq 0} I_{n}\left(f_{n}\right)$ with and $I_{0}\left(f_{0}\right)=E(F)$ where the series converges in $L^{2}$

For $\phi_{1}, \cdots, \phi_{n} \in \mathcal{H}$, let $F=g\left(B^{H}\left(\phi_{1}\right), \cdots, B^{H}\left(\phi_{n}\right)\right)$ with $g$ smooth compactly supported. Then Malliavin derivative $D$ is $\mathcal{H}$ valued random variable defined as follows:

$$
D F=\sum_{i=1}^{n} \frac{\partial g}{\partial x_{i}}\left(B^{H}\left(\phi_{1}\right), \cdots, B^{H}\left(\phi_{n}\right)\right) \phi_{i} .
$$

If $\mathcal{H}$ is $L^{2}(\mathbb{R})$ for some non atomic measure then $D F$ can be identified as follows: $D F=\left(D_{t} F\right)_{t \in \mathbb{R}}$

$$
D_{t} F=\sum_{i=1}^{n} \frac{\partial g}{\partial x_{i}}\left(B^{H}\left(\phi_{1}\right), \cdots, B^{H}\left(\phi_{n}\right)\right) \phi_{i}(t), t \in \mathbb{R}
$$

If $F=I_{n}(f), f \in \mathcal{H}^{\odot n}$, for every $t \in \mathbb{R}$, then

$$
D_{t} F=D_{t} I_{n}(f)=n I_{n-1} f(., t) .
$$

$I_{n-1}(f(., t))$ means $n-1$ multiple stochastic integral is taken with respect to first $n-1$ variables $t_{1}, \cdots, t_{n-1}$ of $f\left(t_{1}, \cdots, t_{n-1}, t\right), t$ is kept fixed. For Malliavin calculus details, see Nualart (1995), Nourdin (2012). To prove asymptotic normality we will use the following two theorems [5.1] and [5.2] taken from Tudor C.A. (2008):

Theorem 8.1. Let $I_{n}(f)$ be a multiple integral of order $n \geq 1$ with respect to an isonormal process M. Then

$$
d\left(\mathcal{L}\left(I_{n}(f)\right), \mathcal{N}(0,1)\right) \leq c_{n}\left[E\left(\left|D I_{n}(f)\right|_{\mathcal{H}}^{2}-n\right)^{2}\right]^{\frac{1}{2}}
$$

where $D$ is the Malliavin derivative with respect to $B^{H}$ and $\mathcal{H}$ is the canonical Hilbert space associated to $B^{H}$. Here $d$ can be any of the distances like Kolmogorov Smirnov distance, or total variation distance etc. and depending upon $d$ and the order $n$ one will end up a constant $c_{n} . \mathcal{L}\left(B^{H}\right)$ stands for law of $B^{H}$.

Theorem 8.2. Fix $n \geq 2$ and let $\left(F_{k}, k>1\right.$ ), $F_{k}=I_{n}\left(f_{k}\right)$ (with $f_{k} \in \mathcal{H}^{\odot n}$, for every $k \geq 1$ ) be a sequence of square integrable random variables in the $n$th Wiener chaos of an isonormal process $B^{H}$ such that $E\left[F_{k}^{2}\right]^{2} \rightarrow 1$ as $k \rightarrow \infty$. Then the following are equivalent: (i) The sequence $\left(F_{k}\right)_{k \geq 0}$ converges in distribution to the normal law $\mathcal{N}(0,1)$. (ii) One has $E\left[F_{k}^{4}\right] \rightarrow 3$ as $k \rightarrow \infty$. (iii) For all $1 \leq l \leq n-1$ it holds that $\lim _{k \rightarrow \infty}\left|f_{k} \otimes_{l} f_{k}\right|_{\mathcal{H} \otimes 2(n-l)}=0$. (iv) $\left|D F_{k}\right|_{\mathcal{H}}^{2} \rightarrow n$ in $L^{2}$ as $k \rightarrow \infty$, where $D$ is the Malliavin derivative with respect to $B^{H}$.

The above theorems we will use to prove asymptotic normality for our proposed estimator. 


\section{REFERENCES}

[1] Avellaneda, M., Levy, A. and Pars, A. (1995), "Pricing and hedging derivative securities in markets with uncertain volatilities" Applied Mathematical Finance, 2(2), 73-88.

[2] Barndorff-Nielsen, O. E. and Shephard, N. (2004), "Power and Bipower Variation with Stochastic Volatility and Jumps", Journal Of Financial Econometrics, 2 (1), 1-37.

[3] Biagini, F., ksendal, B., Sulem, A. and Wallner, N. (2004), "An introduction to white-noise theory and Malliavin calculus for fractional Brownian motion", Proc. Royal Society London, 460, 347-372.

[4] Biagini, F., Hu,Y., Oksendal,B. and Zhang, T. (2008), "Stochastic Calculus for Fractional Brownian Motion and Applications, Springer.

[5] Bjrk, T. and Hult, H. (2005), "A note on Wick products and the fractional Black-Scholes model", Finance and Stochastics, 9(2), 197-209.

[6] Breton, J. et al. (2009), "Exact confidence intervals for the Hurst parameter of a fractional Brownian motion", Electronic Journal of Statistics 3, 416-425.

[7] Cheridito, P. (2003), "Arbitrage in fractional Brownian motion models" Finance and Stochastics 7 (4), $533-553$.

[8] Cont, R. (2005), "Long range dependence in financial markets", In J. Lvy-Vhel \& E. Lutton (Eds.), Fractals in engineering: New trends in theory and applications (pp. 159-180): Springer

[9] Elliott, R.J., van der Hoek, J. (2003), "A general White noise theory and applications to finance", Mathematical Finance 13, 301330.

[10] Guasoni, P. (2006), "No arbitrage under transaction costs, with fractional Brownian motion and beyond", Mathematical Finance 16 (3), 569-582.

[11] Mandelbrot, B. B. (1997), "Fractals and scaling in finance, discontinuity, concentration, risk", Springer.

[12] Mykland, P. A. (2000), "Conservative delta hedging", Annals of Applied Probability, 10(2), 664-683.

[13] Necula,C. (2008), "Option Pricing in a Fractional Brownian Motion Environment", Advances in Economic and Financial Research - DOFIN Working Paper Series 2, Bucharest University of Economics, Center for Advanced Research in Finance and Banking - CARFIB.

[14] Nourdin, I. (2008), "Asymptotic behavior of weighted quadratic and cubic variations of fractional brownian motion", Annals of probability, 36(6), 2159-2175.

[15] Nourdin, I. and Reveillac, A. (2009), "Asymptotic Behavior Of Weighted Quadratic Variations Of Fractional Brownian Motion: The Critical Case H =1/4", Annals of Probability, 37(6), 2200-2230.

[16] Nourdin, I., (2012), "Selected aspects of fractional Brownian motion", Springer.

[17] Nualart, D., (1995), "Malliavin calculus and related topics", Springer.

[18] Prakasa Rao, B.L.S. (2010), "Statistical Inference for Fractional Diffusion Processes", Wiley.

[19] Rogers, L.C.G. (1997), "Arbitrage with fractional Brownian motion", Mathematical Finance, 7, 95-105.

[20] Shiryaev, A. N. (1999), "Essentials of stochastic finance: facts, models, theory", World Scientific.

[21] Tudor, C. A., (2008), "Analysis of variance for self-similar processes", Springer.

[22] Xiao,W., Zhang, W. and Zhang, X. (2013), "Parameter identification for the discretely observed geometric fractional Brownian motion", Journal of Statistical Computation and Simulation, 85(2), 269-283.

[23] Zhang, L. et al. (2005), "A tale of two scales: determining integrated volatility with noisy high frequency data", Journal of American Statistical Association , 100(472), 1394-1411. 